\title{
A clinically relevant blunt spinal cord injury model in the regeneration competent axolotl (Ambystoma mexicanum) tail
}

\author{
MATHIAS MØLLER THYGESEN ${ }^{1,2}$, HENRIK LAURIDSEN ${ }^{1}$, MICHAEL PEDERSEN $^{1}$, \\ DARIUSZ ORLOWSKI ${ }^{3}$, TRINE WERENBERG MIKKELSEN ${ }^{3}$ and MIKKEL MYLIUS RASMUSSEN ${ }^{2}$ \\ ${ }^{1}$ Comparative Medicine Lab, Institute of Clinical Medicine, Aarhus University, 8200 Aarhus N; \\ ${ }^{2}$ Department of Neurosurgery, Aarhus University Hospital; ${ }^{3}$ Center for Experimental Neuroscience (CENSE), Institute of \\ Clinical Medicine, The Department of Neurosurgery, Aarhus University Hospital, 8000 Aarhus C, Denmark
}

Received August 23, 2018; Accepted January 16, 2019

DOI: $10.3892 / \mathrm{etm} .2019 .7193$

\begin{abstract}
A randomized controlled and blinded animal trial was conducted in the axolotl (Ambystoma mexicanum), which has the ability to regenerate from transectional spinal cord injury (SCI). The objective of the present study was to investigate the axolotl's ability to regenerate from a blunt spinal cord trauma in a clinical setting. Axolotls were block-randomized to the intervention $(n=6)$ or sham group $(n=6)$. A laminectomy of two vertebrae at the level caudal to the hind limbs was performed. To induce a blunt $\mathrm{SCI}$, a $25 \mathrm{~g}$ rod was released on the exposed spinal cord. Multiple modalities were applied at baseline (pre-surgery), and subsequently every third week for a total of 9 weeks. Gradient echo magnetic resonance imaging (MRI) was applied to assess anatomical regeneration. To support this non-invasive modality, regeneration was assessed by histology, and functional regeneration was investigated using swimming tests and functional neurological examinations. MRI suggested regeneration within 6 to 9 weeks. Histological analysis at 9 weeks confirmed regeneration; however, this regeneration was not complete. By the experimental end, all animals exhibited restored full neurological function. The present study demonstrated that the axolotl is capable of regenerating a contusion SCI; however, the duration of complete regeneration required further investigation.
\end{abstract}

\section{Introduction}

Spinal cord injury (SCI) is associated with severe morbidity in terms of neurological disability and has major impact on

Correspondence to: Mr. Mathias Møller Thygesen, Comparative Medicine Lab, Institute of Clinical Medicine, Aarhus University, Building G, Palle Juul-Jensens Boulevard 99, 8200 Aarhus N, Denmark

E-mail:matthy@clin.au.dk

Key words: spinal cord injuries, ambystoma mexicanum, regeneration, magnetic resonance imaging, histology quality of life, due to impaired bladder and bowel control (1-3). The most prevalent causes of SCI are traffic accidents and falls, and they generally occur as blunt trauma $(4,5)$.

Although grafting of tissue from peripheral nerves to CNS lesions has unlocked some regenerative potential in mammals, the human organism is incapable of restoring neural tissue following SCI (6-8). On the contrary, the Mexican axolotl (Ambystoma mexicanum), a widely used model in regenerative biology, has the ability to regenerate from transection of the spinal cord (9-12). Literature has reported different durations of successful regeneration, ranging between 7 days and 23 months (9-11).

However, SCI models in the axolotl are traditionally performed as spinal cord transection (or direct tail amputation), thus the novelty in this study is in the application of a more clinically relevant type of injury, namely a blunt contusion injury. To our knowledge, no studies on blunt SCI in the axolotl have been performed though this type of injury reflects a more clinically relevant situation than transection (9-12).

In this proof of concept study, we demonstrate anatomically and functionally that the axolotl is capable of regenerating a clinically relevant SCI. In line with the scientific tradition, it will then be the purpose of subsequent studies to reveal the molecular mechanisms behind this phenomenon.

In conclusion, the purpose of the present study was to investigate the axolotl's ability to regenerate from a blunt contusion trauma.

\section{Materials and methods}

Animals and anesthesia. Animals used in the present study were Mexican axolotls (Ambystoma mexicanum) (mean body mass \pm STD: $12.12 \mathrm{~g} \pm 1.25 \mathrm{~g}$ ) obtained from a commercial breeder (Exoterra GmbH, Holzheim, Germany). Animals were housed individually in plastic containers with a $10 \mathrm{~cm}$ water depth and a $930 \mathrm{~cm}^{2}$ surface area with regular water change and a 12 h:12 h light:dark cycle. They were fed every other day with protein enriched trout pellets. Anesthesia was obtained using $200 \mathrm{mg} / \mathrm{l}$ ethyl-4-aminobenzoate.

Overview. A randomized blinded controlled trial study was performed, involving 6 animals in both a surgery and sham 
group. The model was designed as a surgical intervention, with the aim of inducing a blunt trauma to the spinal cord. Furthermore, all data were blinded with respect to time.

All animals were examined using high-field magnetic resonance imaging (MRI), including diffusion tensor imaging (DTI). Furthermore behavioral modalities such as neurological examinations, and swimming tests were performed as well. All animals were sacrificed for histology at 9 weeks post injury (WPI). Three additional animals (2 SCI, and 1 sham) were sacrificed immediately after surgery for histology.

All modalities were applied at baseline (pre-surgery). MRI protocols were applied immediately after surgery. For follow-up, MRI and all other modalities were applied every third week for 9 weeks.

Surgery. The animals were randomized to the SCI and sham groups though ensuring similar group size, with the project investigators being blinded. To induce a controlled spinal cord contusion trauma, the animals underwent laminectomy of two adjacent vertebral levels, caudal to the hind limbs. Under microscopic magnification and with the animal in prone position, transection through the keel to the spinal process midline was performed and extended into bilateral horizontal sections. The spinal processes and laminae were exposed. The vertebrae in the axolotl are of the opisthocoelous type. A laminectomy was performed with a bilateral micro-scissor cut, and the laminae elevated using forceps, leaving the underlying dura and spinal cord exposed (Fig. 1).

For the intervention group, a standardized trauma unit was designed (Fig. 1). The equipment allowed the rod of a $25 \mathrm{~g}$ weight to fall exactly $30 \mathrm{~mm}$ onto the exposed spinal cord, transferring a theoretical $7.4 \mathrm{~mJ}$ of energy directly to the spinal cord. These values were decided upon from extensive piloting. During piloting we titrated to the lowest falling height and weight which produced disruption of the dorsal artery and hence macroscopical bleeding. Literature has reported no previous standards for this given trauma. For the sham group, all above-mentioned steps were performed, with exception of the spinal cord trauma.

Observations during the first 3 weeks. To ensure wound closure and hinder additional injury, the animals were only observed passively for the first 3 weeks. Animals were not disturbed, and water was changed gently using a pump.

Histology. Tissue samples were fixed in $4 \%$ neutral buffered formaldehyde solution, and decalcified using $2 \mathrm{M} \mathrm{HCl}$ for $1 \mathrm{~h}$ before being processed in a Citadel Shandon 2000 tissue processor (Thermo Fisher Scientific, Inc., Waltham, MA, USA) and embedded in paraffin. The tissue blocks were sectioned into $20 \mu \mathrm{m}$ sections to yield longitudinal sections of the spinal cord. Sections were deparaffined in xylene and rehydrated in decreasing alcohol concentrations and then water. Staining was performed using Mayers hematoxoxylin for $10 \mathrm{~min}$ followed by a $10 \mathrm{~min}$ rinse in tap water, and alcoholic eosin $(0.2 \%$ eosin in $80 \%$ ethanol) for $2 \mathrm{~min}$. Sections were dehydrated through increasing alcohol concentrations, cleared in xylene and closed with Depex.
Anatomical MRI. Anatomical images of the injury were obtained using a gradient echo 3D protocol using an Agilent 9.4 T MRI system and following parameters: TE: $2.24 \mathrm{~ms}$, TR: 4.44 ms, FOV: 50 x50x50 mm m $^{3}$ matrix: 256x256x256 yielding isotropic voxels of $0.2 \mathrm{~mm}$, and averages: 4 . One scan was made at baseline (-0 WPI), one immediately after surgery (+0 WPI), and then at 3 weeks intervals, for 9 weeks (3, 6 and 9 WPI).

MRI images were described in a blinded manner using simple dichotomous outcomes ('yes' or 'no') for each parameter. Continuity of the spinal cord was assessed. Edema of the spinal cord was defined as a lack of visual cerebrospinal fluid (CSF) around the spinal cord, due to displacement of the CSF by the edematous spinal cord.

As a quality assessment, the images were analysed for complete transections, penetrating bone fragments and/or dislocation of the spinal cord as well as post-surgical sequelae incorporating artefacts such as hemosiderin depositions leading to conditions that would make evaluation of the spinal cord impossible.

Neurological examination. The animals were examined neurologically every third week post-surgery. The tail caudal to the injury was stimulated with both tactile (gentle touch with forceps) and nociceptive stimuli (forceps pinching). For both stimuli, 3 attempts were conducted, with the maximum response defining the score of the test: 0 point: No response. 1 point: Local tail movement. 2 points: Truncal movement. 3 points: Coordinated movement of limbs and/or head alongside with truncal movement. 4 points: Animals with immediate coordinated fast movement.

Swimming capacity test. At baseline and every third week from surgery, animals were tested for swimming capacity in a swimming respirometer using a gradual increase of $50 \mathrm{rpm} / 5 \mathrm{~s}$, starting at $200 \mathrm{rpm}$. The maximum swimming capacity was then defined as the moment when the animal would not produce sufficient force to withhold against the water current. All data points were normalized to the baseline mean for the given group yielding a relative measure for water current.

Statistics. Statistical test of the swimming capacity was performed as student's t-test. P-values below 5\% were considered significant. For the neurological score a non-parametric one-tailed Mann-Whitney U test with a critical value of 5 was performed.

Ethical. We certify that all applicable institutional and governmental regulations concerning the ethical use of animals were followed during the course of the present study. The study was conducted under the Approval ID: 2015-15-0201-0061 by the Danish Animal Experiment Inspectorate.

\section{Results}

General observations. One sham group animal was sacrificed 7 days after surgery due to insufficient wound closure. For the remaining animals the wound was closed 2-3 WPI.

Histology. The Hemotoxylin-eosin stained tissue morphology analyzed directly post-surgery (+0 WPI) showed that the 
A

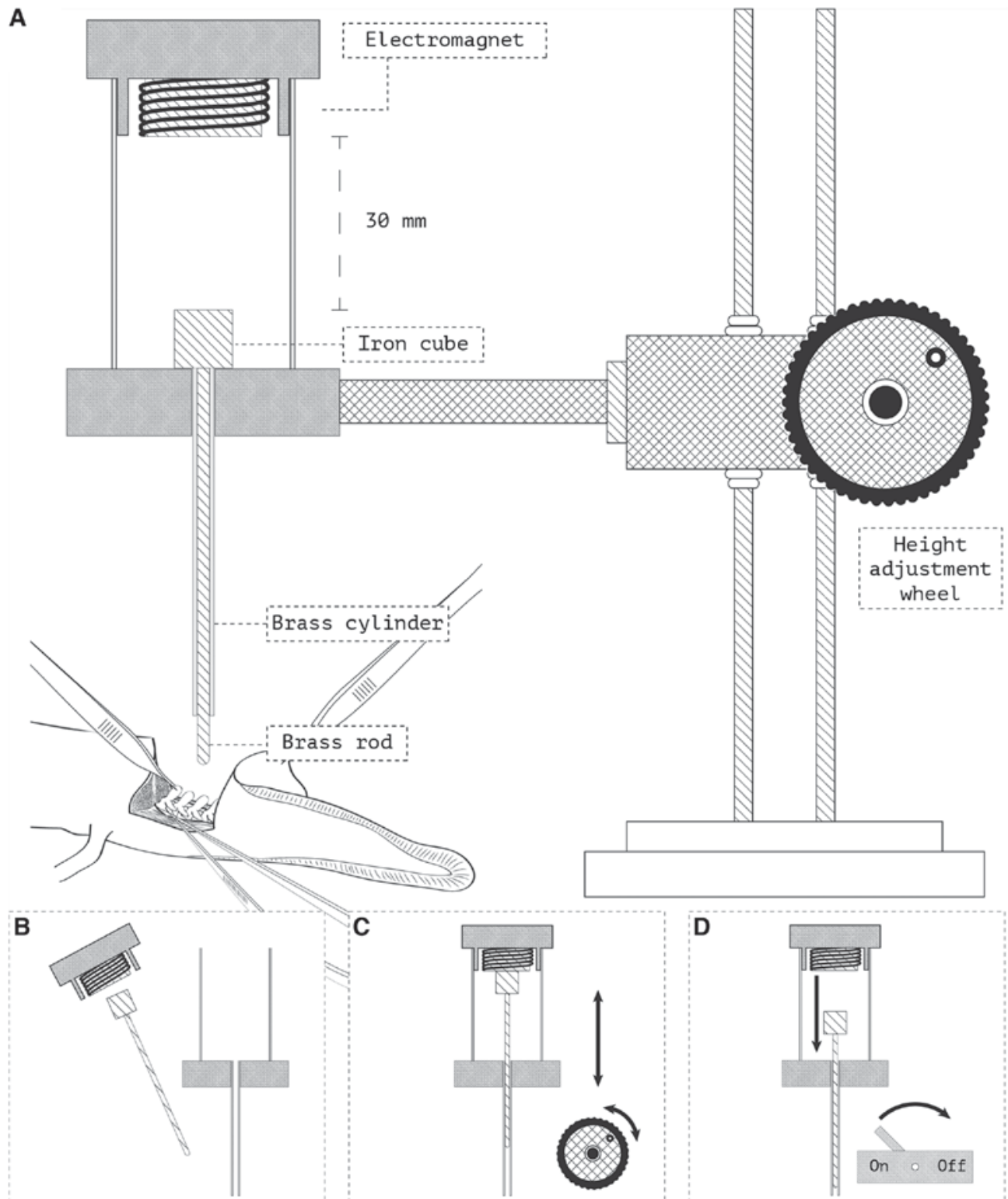

Figure 1. Trauma unit and surgical illustration. (A) Illustration of the trauma unit. The table is 2-way adjustable in the horizontal plane. The design ensures no-touch technique on the animal itself. The animal is fixated on the table using wetted cloth, ensuring that fixation does to inflict damage. (B) The falling rod is connected to the electromagnet, before loading the complex into the brass cylinder. (C) The entire falling mechanism is adjusted, ensuring that the cylinder is placed just above the spinal cord. (D) The falling rod is released by turning of the electromagnet.

intervention group had severe injuries on their spinal cord i.e., the central canal was discontinued (Fig. 2A-1). The tissue surrounding the injury site was edematous and thickened. The sham animals had intact spinal cords, suggesting that surgery did not inflict damage. We observed no signs of edema or infiltration (Fig. 2C-1).

At 9 WPI, some differences in spinal cord morphology between sham and intervention animals were observed. The spinal cords of the sham animals were comparable to the samples acquired post-surgery (+0 WPI), i.e. not showing any signs of discontinuity, thickening, edema or infiltration (Fig. 2D-1). In contrast, the intervention animals showed ependymal proliferation and a compressed spinal cord. The spinal cord and central canal were both found continuous. Organization of the cells was different in the regenerating spinal cord where cells with small nuclei and cell bodies dominate, possibly microglial/macrophages cells (Fig. 2B-1).
Anatomical MRI. At baseline, all animals were blind-scored for continuity and potential signs of injury (Figs. 3A and 4). Eight of the immediate post-surgical (+0 WPI) scans were impossible to describe in a satisfactory manner due to artefacts and hemosiderin deposits. In the remaining three +0 WPI scans, edema was observed in the SCI group and not in the sham group. At 3 WPI, all SCI animals were described and showed full continuity, but still spinal cord edema was observed. MRI from two animals in the sham group suggested edema as well, with the remaining being comparable to baseline. At 6 WPI, MRI of the SCI group was comparable to baseline, except for one animal. In the sham group two animals had findings suggesting edema. Only one of these animals was consistent with findings at 3 WPI. At 9 WPI, the edema was observed in two animals in the SCI group. Edema that was not identified in earlier MRI scans was found in one of the sham group animals. 




Figure 2. Histology. Histological, sagittal sections of spinal cords. (A-1) SCI animal at +0 WPI, (B-1) SCI animal at 9 WPI, (C-1) Sham animal at +0 WPI and (D-1) Sham animal at 9 WPI. Red square: The area of injury for the SCI animals, and the area of laminectomy for the sham animals. Montage of photographs taken using x1.25 objective, scale bars of larger images 1,000 $\mu \mathrm{m}$ and of smaller images $250 \mu \mathrm{m}$. (A-2, B-2, C-2 and D-2) All show the marked area at magnification, x5. Blue arrow: Uninjured spinal cord. SCI, spinal cord injury; WPI, weeks post injury.

Swimming capacity test. The swimming capacity test did not at any time point show significant differences between the sham and intervention groups (Fig. 3B). However, we observed that mean swimming capacity was lower in the SCI group at 3 WPI $(\mathrm{P}=0.34)$. Data revealed a significant difference (lower) swimming capacity at baseline compared to the last follow up for all animals $(\mathrm{P}=0.0008)$.

Neurological examination. When pinching the tail immediately after surgery in pilot experiments we saw subtle clonus-like tail movements. Tactile examination at 3 WPI yielded a non-significant difference of the mean score between the groups (mean difference $=2$ points, U-statistic: $\mathrm{SCI}=6.5$, Sham=12.5). This difference was diminished at the later time points (Fig. 3C). Considering the nociceptive part of the examination, we found no difference at any time point. All animals except one SCI animal at 3 WPI (score: 2) scored 4 points throughout the entire experiment.

\section{Discussion}

The present study demonstrates that the axolotl is capable of morphologically regenerating the spinal cord following a contusion trauma. However, we found that regeneration was incomplete after 9 weeks. Bone regeneration seemed to be limited in our model, which might be a consequence of the critical size defect phenomenon; a phenomenon where bone injury size exceeds a given threshold and the regenerative mechanism is not sufficiently stimulated (13).

The axolotl regenerates by formation of terminal vesicles derived from ependymal cells that proliferate and migrate to the injury site and re-establish contact in the spinal cord $(9,14)$. Axons sprout and grow across the injury, innervating the caudal segments (10). Hence, the axolotl spinal cord is permissive of both axonal regrowth but also of ependymal cell proliferation and glial tissue regeneration. In a study of larvae of a related species, A. maculatum, applying $2 \mathrm{~mm}$ ablations, 

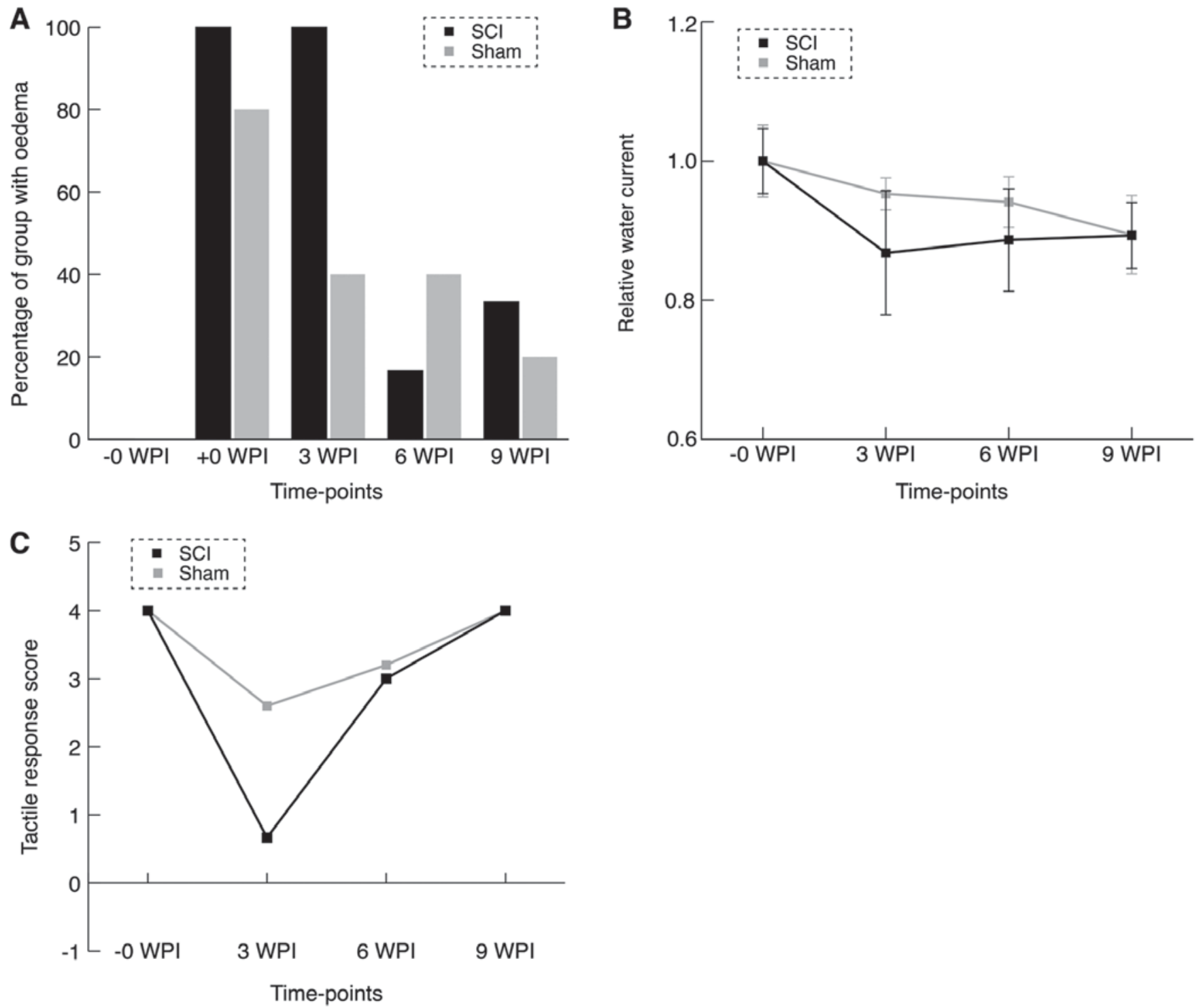

Figure 3. Graphs of MRI, swimming capacity and tactile response. (A) Plot illustrating the results from the MRI scans. For each group the percentage of animals with spinal cord oedema at the given time-points. (B) Average relative swimming capacity. Sham. Normalised RPM, rotations per minute of the water current generating propel. Error bars: 95\% CI. (C) Average tactile neurological examination score. Error bars: 95\% CI. MRI, magnetic resonance imaging.

histology showed regeneration 40 days post injury. All animals had regained gait using their hind limbs, but only half of the animals their ability to use their tail. This finding suggests a functional cranio-caudal regrowth, supporting the view of glial tissue regeneration from both stumps, allowing for axonal regrowth from the cranial stump (9).

The human inability to regenerate the central nervous system is multi-factorial. Among them is Chondroitin sulphate, which mechanically restricts regrowth (15). Blood-derived monocytes and macrophage polarization also inhibit regeneration (16). Myelin associated factors like Nogo-A inhibit axonal sprouting (17,18). Paradoxically, Nogo-A and its receptor have been shown to be part of the axolotl genome (19). All these steps are somehow overcome in the axolotl.

Krogh's principle states that: 'For such a large number of problems there will be some animal of choice or a few such animals on which it can be most conveniently studied'. In accordance with Krogh's principle, this model can facilitate research in spinal cord regeneration mechanisms itself, but also work to test potential inhibitors of regeneration. Increasing levels of potential mammalian inhibitory factors should be able to terminate regeneration, negatively mirroring mammalian studies. This kind of translational approach has been conducted for miRNA $125 \mathrm{~b}$. In the axolotl miRNA $125 \mathrm{~b}$ was found to regulate regeneration of the spinal cord (10). Increasing the levels of miRNA $125 \mathrm{~b}$ in a rodent SCI model increased behavioural scores post-surgery (10). Application of our model in such experiments will enhance the translational value due to a more clinical relevant trauma mechanism. Intervention studies should address the effects of modulating levels of myelin associated inhibitory factors. Additionally, manipulation of chondroitin sulphate, astrocytes and macrophages would be of interest for understanding of the regulatory mechanisms, since these serve in inhibiting SCI progression and regeneration in mammals $(15,16,20)$.

The presence of increased number of ependymal cells in the SCI group as well as the compressed morphology of the spinal cord indicate that regeneration was not complete 


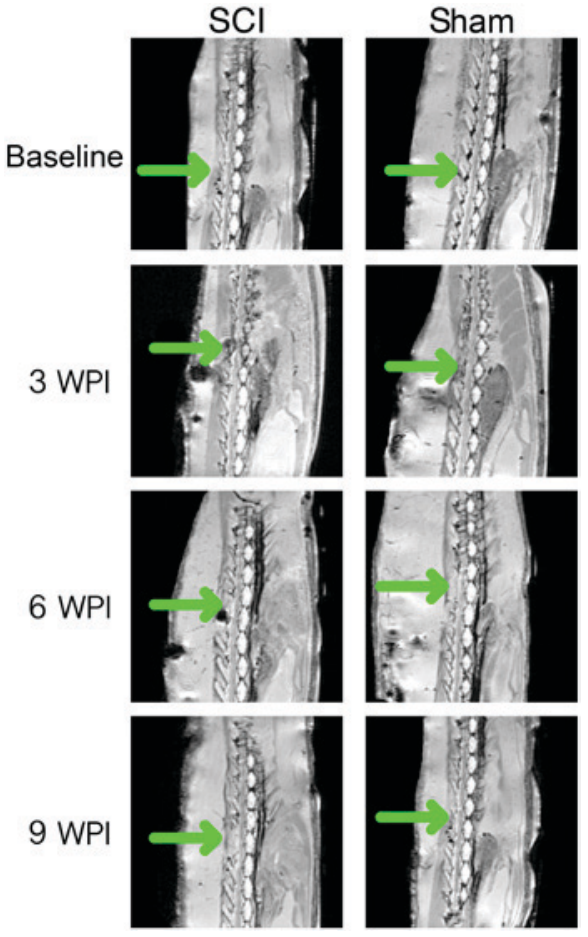

Figure 4. Anatomical MRI scans from various time-points. For each time point representative scans are shown. Do notice that the changes observed are very subtle. The white line of pixels surrounding the spinal cord is CSF. Notice the lack of CSF especially at 3WPI for the SCI animal. On this scan darkening of the spinal cord itself is seen as well. The green arrow marks the area of laminectomy. Scans obtained immediately after surgery are not shown, due to low quality. MRI, magnetic resonance imaging; CSF, cerebrospinal fluid; WPI, weeks post injury.

at 9 weeks ( 9 WPI). Furthermore, the presence of possible microglial cells needs further investigation.

We did not produce immunohistochemistry sections, since the study aimed to describe complete morphological regeneration of the spinal cord (including the central-canal, ependymal layers and vasculature), and not solely axonal regeneration. Future intervention studies could implement axonal staining for beta-III-tubulin, to visualize axonal regeneration in detail.

There seems to be a discrepancy between neurological and morphological outcomes, as all animals at final follow-up seems to be neurological intact. Perhaps our methods were not refined enough to identify small remaining differences. We may as well have had benefitted from a longer timeframe to final follow-up. This finding may reflect that the contusion trauma leaves a less perfect environment for regeneration than surgical transection.

Though non-significant, the neurological tactile examination showed a difference at 3 weeks, which diminished at later time points, which was in coherence with our expectations.

The swimming capacity and nociceptive tests did not show differences between groups. The results support the conclusion that substantial regeneration of neuronal connections happens before 3 WPI.

Interestingly, neither group restored full swimming capacity, suggesting that regeneration of other tissue (e.g. bone and muscle) might be the limiting factor after 3 WPI. The keel of the animals did not regenerate perfectly, maybe due to damages being below critical size defect. This certainly changed the hydrodynamics of the tail and probably the swimming capacity.

Since some of our results yielded insignificant differences between groups, a type II error cannot be excluded; hence a more fine-tuned scale or more replicates might have picked up smaller differences.

The tactile and nociceptive neurological examinations are observer dependent modalities. Both modalities will be dependent on the force applied for both stimuli, which in turn will increase variance on the estimate. However, the study was designed as a randomized and fully blinded study, which would make type-I-errors very unlikely to arise from these potential inaccuracies. Secondly the examinations were performed by the same person throughout the study, eliminating inter-observer variance.

The results of the scans seem prone to noise in the analysis. Some animals, that did not have edema, showed edema at later time points. The chosen protocol does not itself visualize edema, but rather visualizes the lack of CSF fluid. This makes the protocol a surrogate marker edema.

The clonus-like movements observed have been reported in other SCI studies on salamanders $(9,21)$. We interpret these as enhanced spinal reflexes similar to those known from human SCI.

The trauma mechanism and force was chosen based on our extensive pilots. During piloting we found a dose-response between force and neurological impairment. This was to be expected, from our knowledge on SCI in general. However, this study aimed to establish the model and proof-of-concept. Spinal cord contusions are a force dependent condition, and future model development describing the dose-response systematically could be of great interest. Unfortunately, no validated or conventional scale of SCI severity exist for axolotls, which leaves a need for development of such a tool, before commencing the work on a dose-response study.

The axolotl is capable of regenerating a contusion SCI in a randomized, investigator blinded study. The duration of complete regeneration needs to be further investigated.

\section{Acknowledgements}

The authors would like to thank Associate Professor Peter Agger, post.doc, Aarhus University: For his expertise and time on developing the MRI protocols and Associate Professor Steffen Ringgard, post.doc, Aarhus University: For his expertise and time on developing the MRI protocols.

\section{Funding}

The present study was funded by the Riisfort foundation (Grant no. 01-09-2015).

\section{Availability of data and materials}

Data are available on from the corresponding author on reasonable request.

\section{Authors' contributions}

MMT, undertook project initiation, running and piloting the experiments, data analyses, and was responsible for the study 
and drafting of the manuscript. HL was responsible for supervising and performing the experiments, statistical analyses, and reviewing the manuscript. MP supervised the experiments performed the calculations and reviewed the manuscript. DO undertook the piloting and development of the histological analyses, analyses of histological sections and reviewing the manuscript. TWM made the histological sections, performed the experiments and reviewed the manuscript. MMR was the main supervisor, iniated the project, piloted and ran the experiments, and performed the final review of the manuscript.

\section{Ethics approval and consent to participate}

The present study was conducted under the approval id: 2015-15-0201-0061 by the Danish Animal Experiment Inspectorate. We certify that all applicable institutional and governmental regulations concerning the ethical use of animals were followed during the course of the present study.

\section{Patient consent for publication}

Not applicable.

\section{Competing interests}

The authors declare that they have no competing interests.

\section{References}

1. Shavelle RM, DeVivo MJ, Brooks JC, Strauss DJ and Paculdo DR: Improvements in long-term survival after spinal cord injury? Arch Phys Med Rehabil 96: 645-651, 2015.

2. Hicken BL, Putzke JD and Richards JS: Bladder management and quality of life after spinal cord injury. Am J Phys Med Rehabil 80: 916-922, 2001.

3. Levi R, Hultling C, Nash MS and Seiger A: The Stockholm spinal cord injury study: 1 . Medical problems in a regional SCI population. Paraplegia 33: 308-315, 1995.

4. Bjørnshave Noe B, Mikkelsen EM, Hansen RM, Thygesen M and Hagen EM: Incidence of traumatic spinal cord injury in Denmark, 1990-2012: A hospital-based study. Spinal Cord 53: 436-440, 2015.

5. Singh A, Tetreault L, Kalsi-Ryan S, Nouri A and Fehlings MG: Global prevalence and incidence of traumatic spinal cord injury Clin Epidemiol 6: 309-331, 2014.

6. Aguayo AJ, Rasminsky M, Bray GM, Carbonetto S, McKerracher L, Villegas-Pérez MP, Vidal-Sanz M and Carter DA: Degenerative and regenerative responses of injured neurons in the central nervous system of adult mammals. Philos Trans R Soc Lond B Biol Sci 331: 337-343, 1991.
7. Aguayo AJ, Björklund A, Stenevi U and Carlstedt T: Fetal mesencephalic neurons survive and extend long axons across peripheral nervous system grafts inserted into the adult rat striatum. Neurosci Lett 45: 53-58, 1984.

8. Richardson PM, Issa VM and Aguayo AJ: Regeneration of long spinal axons in the rat. J Neurocytol 13: 165-182, 1984.

9. Butler EG and Ward MB: Reconstitution of the spinal cord following ablation in urodele larvae. J Exp Zool 160: 47-65, 1965.

10. Diaz Quiroz JF, Tsai E, Coyle M, Sehm T and Echeverri K: Precise control of miR-125b levels is required to create a regeneration-permissive environment after spinal cord injury: A cross-species comparison between salamander and rat. Dis Model Mech 7: 601-611, 2014.

11. Clarke JD, Alexander R and Holder N: Regeneration of descending axons in the spinal cord of the axolotl. Neurosci Lett 89: 1-6, 1988.

12. McHedlishvili L, Mazurov V and Tanaka EM: Reconstitution of the central nervous system during salamander tail regeneration from the implanted neurospheres. Methods Mol Biol 916: 197-202, 2012.

13. Hutchison C, Pilote $\mathrm{M}$ and Roy S: The axolotl limb: A model for bone development, regeneration and fracture healing. Bone 40: 45-56, 2007.

14. Lacroix S, Hamilton LK, Vaugeois A, Beaudoin S, Breault-Dugas C, Pineau I, Lévesque SA, Grégoire CA and Fernandes KJ: Central canal ependymal cells proliferate extensively in response to traumatic spinal cord injury but not demyelinating lesions. PLoS One 9: e85916, 2014.

15. James ND, Shea J, Muir EM, Verhaagen J, Schneider BL and Bradbury EJ: Chondroitinase gene therapy improves upper limb function following cervical contusion injury. Exp Neurol 271: 131-135, 2015.

16. Silver J, Schwab ME and Popovich PG: Central nervous system regenerative failure: Role of oligodendrocytes, astrocytes and microglia. Cold Spring Harb Perspect Biol 7: a020602, 2014.

17. Freund P, Wannier T, Schmidlin E, Bloch J, Mir A, Schwab ME and Rouiller EM: Anti-Nogo-A antibody treatment enhances sprouting of corticospinal axons rostral to a unilateral cervical spinal cord lesion in adult macaque monkey. J Comp Neurol 502: 644-659, 2007.

18. Zorner B and Schwab ME: Anti-Nogo on the go: From animal models to a clinical trial. Ann N Y Acad Sci 1198 (Suppl 1): E22-E34, 2010

19. Hui SP, Monaghan JR, Voss SR and Ghosh S: Expression pattern of Nogo-A, MAG, and NgR in regenerating urodele spinal cord. Dev Dyn 242: 847-860, 2013.

20. Wanner IB, Anderson MA, Song B, Levine J, Fernandez A, Gray-Thompson Z, Ao Y and Sofroniew MV: Glial scar borders are formed by newly proliferated, elongated astrocytes that interact to corral inflammatory and fibrotic cells via STAT3-dependent mechanisms after spinal cord injury. J Neurosci 33: 12870-12886, 2013.

21. Chevallier S, Landry M, Nagy F and Cabelguen JM: Recovery of bimodal locomotion in the spinal-transected salamander, Pleurodeles waltlii. Eur J Neurosci 20: 1995-2007, 2004.

This work is licensed under a Creative Commons Attribution-NonCommercial-NoDerivatives 4.0 International (CC BY-NC-ND 4.0) License. 